\title{
Intravenous Diclofenac vs. Tramadol infusion for Post-operative Pain Management: A Randomized Trial.
}

\author{
Thakur Binay ${ }^{1}$, Devkota Mukti ${ }^{1}$, Khanal Krishna ${ }^{2}$, Sapkota Bishal ${ }^{1}$ \\ ${ }^{1}$ Department of Surgical Oncology, Thoracic Oncosurgery unit. B.P. Koirala Memorial Cancer Hospital, Bharatpur, Nepal. \\ ${ }^{2}$ Department of Internal Medicine, INTEGRIS Baptist Medical Center, Oklahoma City, USA.
}

\begin{abstract}
Purpose: The study was conducted to compare the analgesic and adverse effect profiles of intravenous infusion of Tramadol and Diclofenac in post-operative pain management.

Method: Cancer patients undergoing major surgery were prospectively randomized into two groups of 35 and was entered into single blinded clinical trial. Patients in each arm received either Tramadol (Gr A) or Diclofenac Infusion (Voveran $\left.{ }^{\circledR}\right)(G r$ B) for first 48 hours after surgery. Pain intensity was measured based on a 10 point Verbal Rating Scale (VRS). VRS was obtained at 6, 12, 18, 24, 30, 36, 42 and 48 hrs post-operatively.

Result: The mean age was 56.3 and 49.5 years in $\mathrm{Gr} A$ and $B$, respectively $(p=.056)$. Mean length of incision was $16.46 \mathrm{~cm}$ and $18.23 \mathrm{~cm}$ in $\mathrm{Gr} A$ and $B$, respectively $(p=.1)$. On 0 post-operative day, 14 patients in $\mathrm{Gr} A$ and 6 patients in Gr B required accessory analgesics $(p=.18)$. On 1 st post-operative day, 2 patients in $G r A$ and 1 patient in $\mathrm{Gr} B$ required accessory analgesics $(p=.6)$. One patient on Tramadol group had nausea post-operatively. Average cost was 471.73 and 227.80 Nepali rupees in $G r A$ and $G r B$, respectively ( $p=.032$ ).

Conclusion: Intravenous infusion of Diclofenac should be considered as a safe and cheaper alternative to Tramadol infusion for pain management after major surgical oncological procedures.
\end{abstract}

Keywords: Diclofenac, Tramadol, Analgesia.

\section{Introduction}

Pain relief has always been one of the primary aims of Medicine. Post-operative pain is an unpleasant experience for the patient. It is associated with a number of physiological responses contributing to organ dysfunction and post-operative morbidity. Despite great advances in the field of pain management, studies have shown that a significant number of patients still experience moderate or severe post-operative pain. ${ }^{1}$ In 1994, a study conducted in UK found that moderate pain was experienced by $25.2 \%$ of patients and severe pain by $9.2 \%$ of patient post-operatively. ${ }^{2}$ Diclofenac has been successfully administered by intravenous infusion (75 $\mathrm{mg}$ in $100 \mathrm{ml}$ isotonic saline over $30 \mathrm{~min}$ ) after orthopedic surgery without adverse effects. ${ }^{3}$ Infusion of intravenous Diclofenac, a new trend in the field of pain management has been adopted in few European Countries like Finland, France and Turkey. This article seeks to explore some of the issues surrounding the provision of post-operative analgesia.

\section{Methods}

Total number of 70 surgical patients was prospectively randomized into two groups of 35 and was entered into single blinded clinical trial. Each patient received either Tramadol infusion (Group A) or Diclofenac infusion (Group B) for the first 48 hours after surgery. Diclofenac group patients received intravenous infusion of Diclofenac(Voveran ") 2mg/kg/24 hrs (maximum dose $150 \mathrm{mg} /$ day) with $1 \mathrm{ml}$ of $7.5 \% \mathrm{NaHCO}_{3}$ for buffering and patients on Tramadol arm received $12 \mathrm{mg} / \mathrm{hr} / 24 \mathrm{hr}$. The drugs were diluted in $50 \mathrm{ml}$ of normal saline and given

Correspondence

Dr. Binay Thakur MD, PhD, Chief, Thoracic Oncosurgery

B.P.Koirala Memorial Cancer Hospital, Nepal, Tel: 00977-56-525725, binaythakur@hotmail.com 
at $2 \mathrm{ml} / \mathrm{kg} /$ day through infusion pump. When infusion pumps were not available the drugs were diluted in $500 \mathrm{ml}$ of normal saline and were given at 5 drops $/ \mathrm{min} /$ day intravenous infusion. Pain intensity was measured based on a 10 point Verbal Rating Scale (VRS). VRS was obtained preoperatively and at $6,12,18,24,30,36$, 42 and 48 hours post-operatively. In VRS, pain in the range of 1-4 point was categorized as mild, 5-7 point as moderate and 7-10 as severe and data was gathered accordingly. Additional coverage of analgesic effect was achieved with bolus injection of accessory analgesics on patient need basis. After 48 hours of surgery, the same drugs were given by oral route or if oral route had not to be used yet because of the nature of the surgery, bolus intravenous tramadol or pethidine were used as per requirement. We excluded the Patients with hypersensitivity against either of the drugs, h/o allergy to any other NSAID, third trimester of pregnancy, active peptic ulcer hemorrhage, severe cardiac failure, severe hepatic failure, inflammatory bowel disease, and renal failure. Pediatric patients were excluded as well. Side effect profile was maintained based on patient's complaints and physical examination findings. Statistical analysis was done using statistical package for the social science (SPSS) 11.0 software. The patients were analyzed on the basis of Verbal Rating Scale of pain, requirement of accessory analgesics on 0 and $1^{\text {st }}$ post-operative days, side effect profiles and financial burden to them, using SPSS 11.0 software. The research project was approved by the Institutional Review Board.

\section{Results}

There were 70 patients with 35 in each arm. In Tramadol Group (Group A), there were 14 male, 21 female whereas in Diclofenac group (Group B), there were 11 male and 24 female. Average age for Group A was 56.31 yrs and 49.54 yrs for Group B. Mean incision length for Group A was $16.56 \mathrm{~cm}$ and for Group B, it was $18.23 \mathrm{~cm}$. Operating time (mean) was 1 hour and 55 minutes for Group A and 2 hours 4 minutes for Group B. Assessment of disease related pain was done pre-operatively on VRS which has been shown in Table I.

Operative procedures performed in each group have been depicted in the Table II.

Table III shows the VRS assessment of pain on 0 postoperative days.

Patients in Group A needed significantly more accessory analgesics than in Group B on 0 post-operative day and first post-operative day.

Table IV depicts the data about accessory pain control on 0 post-operative days.

On $1^{\text {st }}$ post-operative day, 8 patients in Diclofenac Group had no pain and 5 patients in Tramadol Group had no pain which is depicted in the Table V.

On $1^{\text {st }}$ post-operative day, 2 patients in Tramadol group required accessory analgesics and one patient in Diclofenac Group required accessory analgesic.

Table VI depicts the accessory analgesic data on $1^{\text {st }}$ postoperative day.

Post-operative complications were assessed for each group. No problems were encountered with excessive bleeding in any group. One patient in Tramadol Group had nausea sensation. One patient in each arm had wound gaping. Post-operative complications were managed accordingly.

Cost of Diclofenac was cheaper than Tramadol. Average cost was 471.73 Nepali rupees and 227.80 Nepali rupees in Group A and Group B, respectively ( $\mathrm{p}=.032)$

Table I. Pre-operative disease related pain assessment

\begin{tabular}{|l|l|l|l|}
\hline Severity & Group A & \multicolumn{1}{c|}{ Group B } & p value \\
\hline None & 25 & 22 & \\
\hline Mild & 8 & 8 & \\
\hline Moderate & 1 & 2 & \\
\hline Severe & 1 & 3 & 0.677 \\
\hline
\end{tabular}

Table II. Operative Procedures

\begin{tabular}{|l|c|c|}
\hline Procedures & Group A & Group B \\
\hline Radical mastectomy & 10 & 8 \\
\hline Gastrojejunostomy & 4 & 3 \\
\hline Subtotal gastrectomy & 4 & 4 \\
\hline Lobectomy(Pulmonary) & 3 & 4 \\
\hline Extended cholecystectomy & 4 & 0 \\
\hline Exploratory laparotomy & 2 & 2 \\
\hline Radical groin dissection & 1 & 2 \\
\hline 3-incision esophagectomy & 1 & 2 \\
\hline Hemicolectomy & 1 & 1 \\
\hline Relaparotomy, ileostomy & 0 & 1 \\
\hline Hartman's procedure & 1 & 0 \\
\hline Pulmonar wedge resection & 1 & 0 \\
\hline Mastectomy, TRAM flap & 0 & 2 \\
\hline Excision of retrperitoeanl mass & 0 & 1 \\
\hline
\end{tabular}




\begin{tabular}{|l|l|l|}
\hline Sigmoid colectomy & 1 & 0 \\
\hline Wide local excision & 1 & 0 \\
\hline TAH, BSO, omentectomy & 0 & 4 \\
\hline Ileocolic anastomosis & 0 & 1 \\
\hline THE $^{\dagger}$ & 0 & 1 \\
\hline
\end{tabular}

"Transabdominal hysterectomy, oophoretomy, omentectomy ${ }^{\dagger}$ Transhiatal gastroesophagectomy

Table III. 0 Post-operative day pain.

\begin{tabular}{|l|c|c|}
\hline \multicolumn{1}{|c|}{ Severity } & Group A & Group B \\
\hline No pain & 5 & 4 \\
\hline Mild pain & 28 & 31 \\
\hline Moderate pain & 2 & 0 \\
\hline Severe pain & 0 & 0 \\
\hline
\end{tabular}

$\mathrm{p}=.322$

Table IV. 0 Post-operative day accessory analgesics.

\begin{tabular}{|l|c|c|}
\hline Accessory Analgesic x times & Group A & Group B \\
\hline None & 21 & 29 \\
\hline Tramadol x1 & 1 & 0 \\
\hline Pethidinex1 & 7 & 5 \\
\hline Pethidinex2 & 1 & 0 \\
\hline Diclofenac & 5 & 1 \\
\hline
\end{tabular}
$\mathrm{p}=.179$

Table V. $1^{\text {st }}$ Post-operative day pain control.

\begin{tabular}{|l|l|l|}
\hline Pain Assessment & Tramadol & Diclofenac \\
\hline No pain & 5 & 8 \\
\hline Mild pain & 30 & 27 \\
\hline Mod/ severe pain & 0 & 0 \\
\hline
\end{tabular}

$\mathrm{p}=.356$

Table VI. $1^{\text {st }}$ Post-operative day accessory analgesics.

\begin{tabular}{|l|c|c|}
\hline Accessory Analgesics x times & Tramadol & Diclofenac \\
\hline None & 33 & 34 \\
\hline Pethidine x1 & 1 & 0 \\
\hline Diclofenac $x 1$ & 1 & 1 \\
\hline
\end{tabular}
$\mathrm{p}=.602$

\section{Discussion}

The emotional distress by a surgical experience is aggravated by the severity of post-operative pain. Pain management after the operation is one of the most important challenges which not only provides certain comforts to the patients but facilitates early mobilization. Post-operative pain is a classic indication for systemic analgesic use. Non steroid anti inflammatory drugs (NSAIDS) are useful for post-operative pain management because surgery causes both pain and inflammation. Diclofenac has a low to moderate preference to block the COX-2 isoenzyme (approximately 10 fold) and said to have somewhat lower incidence of gastrointestinal complaints than noted with Indomethacin or Aspirin. Diclofenac inhibits lipoxygenase pathways, thus reducing formation of the leukotrienes. In addition, it inhibits Phospholipase A2 as part of its mechanism of action. These additional actions may explain the high potency of Diclofenac. Diclofenac is associated with adverse renal effects caused by the reduction in synthesis of renal prostaglandins ${ }^{4}$ in sensitive persons or animal species (vultures), and potentially during long term use in nonsensitive persons if resistance to side effects decreases with age. Use of Diclofenac in animals has been reported to have led to a sharp decline in the vulture population in the Indian subcontinent, up to $95 \%$ in some areas. ${ }^{5}$ The mechanism is probably renal failure, a known side effect of Diclofenac. Government of Nepal, Ministry of Health and Population, Department of Drug Administration has phased out license and import recommendation letter of Diclofenac for veterinary use based on the possible danger to the ecological system since January, $2007 .^{6}$ Diclofenac should be judiciously used in the elderly human, those on ACE (angiotensin converting enzyme) inhibitors, in the presence of hypovolemia, dehydration and hypotension. The study by Campbell et al has shown successful use of intravenous diclofenac without adverse effect after dental surgery. ${ }^{7}$ Some literatures have described low incidence of side effects, particularly with short term exposure, and concern regarding adverse reactions shouldn't be allowed to limit their usefulness in the practice of post-operative pain management. Nevertheless, Diclofenac should be used with great care, if at all, in certain specific circumstances. Diclofenac should be avoided in cases where extensive surgical resection is anticipated. ${ }^{8}$

Diclofenac is used mainly for musculoskeletal complaints, especially arthritis (rheumatoid arthritis, osteoarthritis, spondylarthritis, ankylosing spondylitis), gout attacks, and pain management in case of kidney stones and gall stone. An additional indication is the treatment of acute migraines. Diclofenac is used commonly to treat 
mild to moderate post-operative or post-traumatic pain, particularly when inflammation is also present, and is effective against menstrual pain.

The mode of action of tramadol has yet to be fully understood, but it is believed to work through modulation of the noradrenergic and serotonergic systems in addition to its mild agonism of the $\mu$-opioid receptor. The contribution of non-opioid activity is demonstrated by the analgesic effects of tramadol not being fully antagonised by the $\mu$-opioid receptor antagonist naloxone. The most commonly reported adverse drug reactions are nausea, vomiting, sweating and constipation. Drowsiness is reported, although it is less of an issue than for other opioids. Patients prescribed tramadol for general pain relief along with other agents have reported uncontrollable withdrawallike nervous tremors if weaning off the medication happens too quickly. Respiratory depression, a common side effect of most opioids, is not clinically significant in normal doses. By itself, it can decrease the seizure threshold. When combined with selective serotonin reuptake inhibitor (SSRIs), tricyclic antidepressants, or in patients with epilepsy, the seizure threshold is further decreased. Seizures have been reported in humans receiving excessive single oral doses $(700 \mathrm{mg}$ ) or large intravenous doses $(300 \mathrm{mg})$. Tramadol is associated with the development of a physical dependence and a withdrawl symptoms. ${ }^{9}$ Tramadol causes typical opiatelike withdrawal symptoms as well as atypical withdrawal symptoms including seizures. The atypical withdrawal effects are probably related to tramadol's effect on serotonin and norepinephrin reuptake. Symptoms may include anxiety, anguish, sweating and palpitation. It is recommended that patients physically dependent on pain killers take their medication regularly to prevent onset of withdrawal symptoms and when the time comes to discontinue their tramadol, to do so gradually over a period of time which will vary according to the individual patient and dose and length of time on the drug. ${ }^{10,11,12}$ Though so many side effects of Diclofenac infusion have been described, they appear not to be as severe as the complications of tramadol infusion and we found diclofenac as a wonderful analgesic drug which controlled post operative pain adequately. It didn't show any side effect even though we didn't have to use H2-blockers or proton pump inhibitors. So far use of analgesics in Nepal is concerned, there is not a common consensus or guideline. Concept of patient-controlled analgesia is still not being practiced in Nepal and epidural analgesia is used in selected tertiary care centers only. The usual practice is to use intramuscular diclofenac, tramadol or pethidine. We have tried to change the practice of intramuscular injection to intravenous infusion which is much more convenient for patient. Secondly, we have tried to change the dogma that narcotic drugs are better than NSAIDS after major surgery.

Our results have clearly shown that diclofenac infusion is as effective as tramadol infusion after major surgical procedures. At the same time it contributes less financial burden to patient. One drawback of the diclofenac infusion is that it can not be used intravenously for more than 48 hours and later, the pain had to be controlled by some another analgesics if patient was not allowed orally. But after most of the operations, patients were allowed orally after 48 hours and diclofenac could be allowed orally for additional period of time. Overall, the method of diclofenac infusion is very simple; hence it can be carried out at any set-up of hospital in Nepal. Though a larger number of cases are required to know more about the negative aspects of diclofenac, our results have shown that it is a safe and cheap drug for post operative pain control.

\section{Conclusion}

Diclofenac intravenous infusion appears to be a wonderful, safe and cheap drug and can be recommended for postoperative analgesia after major surgeries for first 48 hours.

\section{Aknowledgement}

All authors are thankful to Novartis India limited for providing 200 ampoules of Diclofenac (Voveran ${ }^{\circledR}$ ) for the study.

\section{Reference}

1. Rieck B, Schwemmlek. Systemic Pain therapy, evaluation from surgeon's point of view. Reg Cancer Treat 1990; 3:122-125.

2. Oates JDL, Snowdon SL, Jayson DWH. Failure of pain relief after surgery. Attitudes of ward staff and patients to post-operative analgesia. Anesthesia1994; 49:755-758.

3. Laitinen J, Nuutinen L, Kiiskila E-L, Freudenthal V, Ranta P, Karvonen J. Comparison of intravenous diclofenac, indomethacin and oxycodone as postoperative analgesics in patients undergoing knee 
surgery. Euro J Anaesth 1991; 9:21-24

4. Brater DC .Renal Effects of Cyclooxygenase-2 selective inhibitors J Pain symptom Manage 2002; 23:15-23.

5. Oaks JL, Gilbert M, Virani MZ, Watson RT, Meteyer CU, Rideout BA et al. Diclofenac residues as the cause of vulture population decline in Pakistan. Nature 2004; 427: 630-3.

6. Drug Bulletin of Nepal, Dec, 2006- March, 2007; 18:30

7. Campbell WI, Kendrick R, Patterson C. Intravenous diclofenac sodium. Anaesthesia 1990; 45:763-6

8. Cashman J N. Non-steroidal anti-inflammatory drugs versus postoperative pain. J R Soc Med 1993; 86(8):464-467.
9. Barsotti C, Mycyk M. Withdrawal syndrome from tramadol hydrochloride. Am J Emerg Med 2003; 21 (1): $87-8$

10. Choong K, Ghiculescu Ra. Iatrogenic neuropsychiatric syndromes. Aust Fam Physician 2008; 37 (8): 627-9

11. Ripamonti C, Fagnoni E, Conno F. withdrawal syndrome after delayed tramadol intake. Am J Psychiatry 2004; 161 (12): 2326-7

12. Senay E, Adams E, Geller A, Inciardi J, Muñoz A, Schnoll S et al .Physical dependence on Ultram (tramadol hydrochloride): both opioid-like and atypical withdrawal symptoms occur". Drug Alcohol Depend 2003; 69 (3): 233-41 